\title{
Research on intuitionistic fuzzy negations
}

\author{
Nora Angelova ${ }^{1}$ and Krassimir T. Atanassov ${ }^{2}$ \\ ${ }^{1}$ Faculty of Mathematics and Informatics, Sofia University \\ 5 James Bourchier Blvd., 1164 Sofia, Bulgaria \\ e-mail: noraa@fmi.uni-sofia.bg \\ 2 Dept. of Bioinformatics and Mathematical Modelling, \\ Institute of Biophysics and Biomedical Engineering, \\ Bulgarian Academy of Sciences \\ 105 Acad. G. Bonchev Str., 1113 Sofia, Bulgaria \\ e-mail: krat@bas.bg
}

Received: 7 March 2021

Revised: 16 June 2021

Accepted: 27 August 2021

\begin{abstract}
In the theories of intuitionistic fuzzy sets and intuitionistic fuzzy logics, there are 54 different negations. Here, we check the relationship between every two of them.

Keywords: Intuitionistic fuzzy negation, Intuitionistic fuzzy implication, Intuitionistic fuzzy pair, Intuitionistic fuzzy set.
\end{abstract}

2020 Mathematics Subject Classification: 03E72.

\section{Introduction}

Following, extending and correcting [17], here we give short remarks on the results related to the intuitionistic fuzzy negations. We give the full list of the intuitionistic fuzzy negations and three tables of the relations between them. Similar research is made for the first time in fuzzy sets theory. The list of relations between separate negations and implications is also given.

In the series of papers $[2,3,5,7-12,12,15,16,18,18-20,22,24,26,27,29-44,46-48,50,51]$, different intuitionistic fuzzy implications and generated by them intuitionistic fuzzy negations have been defined and some of their basic properties have been studied. In some of these publications, some misprints in the formulas of the implications and of the negations have been found over the last years. Here, we give the full list of the corrected intuitionistic fuzzy negations. 
Historically, the first two intuitionistic fuzzy negations were introduced in [6] by K. Atanassov. Fifteen years ago, the first ten implications, described in [10], were constructed as intuitionistic fuzzy analogues of some existing fuzzy implications in literature (see, e.g., [49]. These ten implications generated three negations. The first two of them coincide with the negations from [6]. It is worth noting that a lot of the first ten implications $\left(\rightarrow_{1}, \rightarrow_{4}, \rightarrow_{5}, \rightarrow_{6}, \rightarrow_{7}, \rightarrow_{10}\right)$ generate one particular negation $\left(\neg_{1}\right)$, three implications $\left(\rightarrow_{2}, \rightarrow_{3}, \rightarrow_{8}\right)$ generate another negation $\left(\neg_{2}\right)$, and only implication $\rightarrow_{9}$ generates a unique (for the first batch of ten implications) negation $\left(\neg_{3}\right)$. Later, two other implications also proved to generate that negation $\neg_{3}\left(\rightarrow_{17}, \rightarrow_{21}\right)$.

All currently existing negations are given in Table 1 . We preserve the numeration from [14, $17,52]$.

It is necessary to mention that there is a mistake in the monographs $[17,52]$. There, an implication $\rightarrow_{52}$ was included that later proved to coincide with the implication $\rightarrow_{8}$. By this reason, the fifty third and the fifty fourth implications are now denoted by $\rightarrow_{52}$ and $\rightarrow_{53}$, respectively, and this is mentioned in Table 5, too.

Let everywhere below the intuituinistic fuzzy truth values of the variable $x$ be $x=\langle a, b\rangle$. In [25], these pairs are called Intuituinistic Fuzzy Pairs (IFPs). Below, we will describe the different forms of $\neg x$.

We must mention that in some formulas we use the following functions

$$
\operatorname{sg}(x)=\left\{\begin{array}{ll}
1 & \text { if } x>0 \\
0 & \text { if } x \leq 0
\end{array}, \quad \overline{\operatorname{sg}}(x)=\left\{\begin{array}{ll}
0 & \text { if } x>0 \\
1 & \text { if } x \leq 0
\end{array} .\right.\right.
$$

Table 1. List of the intuitionistic fuzzy negations

\begin{tabular}{|l|l|}
\hline$\neg_{1}$ & $\langle b, a\rangle$ \\
\hline$\neg_{2}$ & $\langle\overline{\operatorname{sg}}(a), \operatorname{sg}(a)\rangle$ \\
\hline$\neg_{3}$ & $\left\langle b, a \cdot b+a^{2}\right\rangle$ \\
\hline$\neg_{4}$ & $\langle b, 1-b\rangle$ \\
\hline$\neg_{5}$ & $\langle\overline{\operatorname{sg}}(1-b), \operatorname{sg}(1-b)\rangle$ \\
\hline$\neg_{6}$ & $\langle\overline{\operatorname{sg}}(1-b), \operatorname{sg}(a)\rangle$ \\
\hline$\neg_{7}$ & $\langle\overline{\operatorname{sg}}(1-b), a\rangle$ \\
\hline$\neg_{8}$ & $\langle 1-a, a\rangle$ \\
\hline$\neg_{9}$ & $\langle\overline{\operatorname{sg}}(a), a\rangle$ \\
\hline$\neg_{10}$ & $\langle\overline{\operatorname{sg}}(1-b), 1-b\rangle$ \\
\hline$\neg_{11}$ & $\langle\operatorname{sg}(b), \overline{\operatorname{sg}}(b)\rangle$ \\
\hline$\neg_{12}$ & $\left\langle b \cdot(b+a), a \cdot\left(b^{2}+a+b . a\right)\right\rangle$ \\
\hline$\neg_{13}$ & $\langle\operatorname{sg}(1-a), \overline{\operatorname{sg}}(1-a)\rangle$ \\
\hline$\neg_{14}$ & $\langle\operatorname{sg}(b), \overline{\operatorname{sg}}(1-a)\rangle$ \\
\hline$\neg_{15}$ & $\langle\overline{\operatorname{sg}}(1-b), \overline{\operatorname{sg}}(1-a)\rangle$ \\
\hline$\neg_{16}$ & $\langle\overline{\operatorname{sg}}(a), \overline{\operatorname{sg}}(1-a)\rangle$ \\
\hline
\end{tabular}

(Continued on next page) 
Table 1 (Continued from previous page)

\begin{tabular}{|c|c|}
\hline$\neg 17$ & $\langle\overline{\mathrm{sg}}(1-b), \overline{\mathrm{sg}}(b)\rangle$ \\
\hline$\neg 18$ & $\langle b \cdot \operatorname{sg}(a), a \cdot \operatorname{sg}(b)\rangle$ \\
\hline$\neg 19$ & $\langle b \cdot \operatorname{sg}(a), 0\rangle$ \\
\hline$\neg_{20}$ & $\langle b, 0\rangle$ \\
\hline$\neg_{21}$ & $\langle\min (1-a, \operatorname{sg}(a)), \min (a, \operatorname{sg}(1-a))\rangle$ \\
\hline$\neg_{22}$ & $\langle\min (1-a, \operatorname{sg}(a)), 0\rangle$ \\
\hline$\neg_{23}$ & $\langle 1-a, 0\rangle$ \\
\hline$\neg_{24}$ & $\langle\min (b, \operatorname{sg}(1-b)), \min (1-b, \operatorname{sg}(b))\rangle$ \\
\hline$\neg_{25}$ & $\langle\min (b, \operatorname{sg}(1-b)), 0\rangle$ \\
\hline$\neg_{26}$ & $\langle b, a \cdot b+\overline{\operatorname{sg}}(1-a)\rangle$ \\
\hline$\neg_{27}$ & $\langle 1-a, a \cdot(1-a)+\overline{\operatorname{sg}}(1-a)\rangle$ \\
\hline$\neg_{28}$ & $\langle b,(1-b) . b+\overline{\operatorname{sg}}(b)\rangle$ \\
\hline$\neg_{29}$ & $\langle\max (0, b \cdot a+\overline{\operatorname{sg}}(1-b)), \min (1, a \cdot(b \cdot a+\overline{\operatorname{sg}}(1-b))+\overline{\operatorname{sg}}(1-a))\rangle$ \\
\hline$\neg 30$ & $\langle a . b, a .(a . b+\overline{\operatorname{sg}}(1-b))+\overline{\operatorname{sg}}(1-a)\rangle$ \\
\hline$\neg 31$ & $\langle\max (0,(1-a) \cdot a+\overline{\operatorname{sg}}(a)), \min (1, a \cdot((1-a) \cdot a+\overline{\operatorname{sg}}(a))+\overline{\operatorname{sg}}(1-a))\rangle$ \\
\hline$\neg 32$ & $\langle(1-a) \cdot a, a \cdot((1-a) \cdot a+\overline{\operatorname{sg}}(a))+\overline{\operatorname{sg}}(1-a)\rangle$ \\
\hline$\neg 33$ & $\langle b \cdot(1-b)+\overline{\mathrm{sg}}(1-b),(1-b) \cdot(b \cdot(1-b)+\overline{\mathrm{sg}}(1-b))+\overline{\mathrm{sg}}(b))\rangle$ \\
\hline$\neg 34$ & $\langle b \cdot(1-b),(1-b) \cdot(b \cdot(1-b)+\overline{\operatorname{sg}}(1-b))+\overline{\operatorname{sg}}(b)\rangle$ \\
\hline$\neg 35$ & $\left\langle\frac{b}{2}, \frac{1+a}{2}\right\rangle$ \\
\hline$\neg 36$ & $\left\langle\frac{b}{3}, \frac{2+a}{3}\right\rangle$ \\
\hline$\neg 37$ & $\left\langle\frac{2 b}{3}, \frac{2 a+1}{3}\right\rangle$ \\
\hline$\neg 38$ & $\left\langle\frac{1-a}{3}, \frac{2+a}{3}\right\rangle$ \\
\hline$\neg 39$ & $\left\langle\frac{b}{3}, \frac{3-b}{3}\right\rangle$ \\
\hline$\neg 40$ & $\left\langle\frac{2-2 a}{3}, \frac{1+2 a}{3}\right\rangle$ \\
\hline$\neg_{41}$ & $\left\langle\frac{2 b}{3}, \frac{3-2 b}{3}\right\rangle$ \\
\hline$\neg 42, \lambda$ & $\left\langle\frac{b+\lambda-1}{2 \lambda}, \frac{a+\lambda}{2 \lambda}\right\rangle$, where $\lambda \geq 1$ \\
\hline$\neg 43, \gamma$ & $\left\langle\frac{b+\gamma}{2 \gamma+1}, \frac{a+\gamma}{2 \gamma+1}\right\rangle$, where $\gamma \geq 1$ \\
\hline$\neg 44, \alpha, \beta$ & $\left\langle\frac{b+\alpha-1}{\alpha+\beta}, \frac{a+\beta}{\alpha+\beta}\right\rangle$, where $\alpha \geq 1, \beta \in[1, \alpha]$ \\
\hline$\neg_{45, \varepsilon, \eta}$ & $\langle\min (1, b+\varepsilon), \max (0, a-\eta)\rangle$, where $\varepsilon, \eta \in[0,1]$ and $\varepsilon \leq \eta<1$ \\
\hline$\neg 46, \lambda$ & $\left\langle\frac{\lambda-a}{2 \lambda}, \frac{a+\lambda}{2 \lambda}\right\rangle$, where $\lambda \geq 1$ \\
\hline$\neg 47, \lambda$ & $\left\langle\frac{b+\lambda-1}{2 \lambda}, \frac{1-b+\lambda}{2 \lambda}\right\rangle$, where $\lambda \geq 1$ \\
\hline$\neg 48, \gamma$ & $\left\langle\frac{1-a+\gamma}{2 \gamma+1}, \frac{a+\gamma}{2 \gamma+1}\right\rangle$, where $\gamma \geq 1$ \\
\hline$\neg 49, \gamma$ & $\left\langle\frac{b+\gamma}{2 \gamma+1}, \frac{1-b+\gamma}{2 \gamma+1}\right\rangle$, where $\gamma \geq 1$ \\
\hline$\neg_{50, \alpha, \beta}$ & $\left\langle\frac{b-1+\alpha}{\alpha+\beta}, \frac{a+\beta}{\alpha+\beta}\right\rangle$, where $\alpha \geq 1, \beta \in[1, \alpha]$ \\
\hline$\neg 51, \alpha, \beta$ & $\left\langle\frac{b-1+\alpha}{\alpha+\beta}, \frac{1-b+\beta}{\alpha+\beta}\right\rangle$, where $\alpha \geq 1, \beta \in[1, \alpha]$ \\
\hline$\neg_{52}$ & $\langle\overline{\operatorname{sg}}(a)+\operatorname{sg}(a) b, a\rangle$ \\
\hline$\neg_{53}$ & $\left\langle\overline{\operatorname{sg}}(a), \frac{a}{1-b}\right\rangle$ \\
\hline
\end{tabular}




\section{Main results}

As a next step in the research on intuitionistic fuzzy negations, here we determine the relations between every two nonparametric negations - see Table 2 and its continuations in Tables 3 and 4 . For this purpose, we use the software tool - IFSTool, developed by Angelova, [1].

In the cell with coordinates $(i, j)$, stays one of the symbols $<,>,=$, or - . The first symbol corresponds to the case $x \rightarrow_{i} y<x \rightarrow_{j} y$, second symbol corresponds to the case $x \rightarrow_{i} y>x \rightarrow_{j} y$, and the third corresponds to $x \rightarrow_{i} y=x \rightarrow_{i} y$ (that is the normal situation), while the fourth symbol corresponds to the case when there is no one of the three possible relations. Here, $1 \leq i, j \leq 53$ except for $42 \leq i, j \leq 51$.

In the research, we eliminate negations $\neg_{42}, \ldots, \neg_{51}$, because they contain parameters and are incomparable to the other negations. An Open problem is to search for the relations among these ten parametric negations and to find what relations hold between them (for the different values of their parameters) and the rest of the (nonparametric) negations.

Table 2. Relations between the nonparametric intuitionistic fuzzy negations $\neg_{1}, \ldots, \neg 15$

\begin{tabular}{|l|lllllllllllllll|}
\hline & 1 & 2 & 3 & 4 & 5 & 6 & 7 & 8 & 9 & 10 & 11 & 12 & 13 & 14 & 15 \\
\hline 1 & $=$ & - & $<$ & $>$ & $>$ & $>$ & $>$ & $<$ & - & $>$ & - & - & $<$ & $<$ & - \\
\hline 2 & - & $=$ & - & - & $>$ & $>$ & - & $<$ & $<$ & - & - & - & $<$ & - & - \\
\hline 3 & $>$ & - & $=$ & $>$ & $>$ & $>$ & $>$ & - & - & $>$ & - & - & $<$ & $<$ & - \\
\hline 4 & $<$ & - & $<$ & $=$ & $>$ & - & - & $<$ & - & $>$ & $<$ & - & $<$ & $<$ & - \\
\hline 5 & $<$ & $<$ & $<$ & $<$ & $<$ & $<$ & $<$ & $<$ & $<$ & $<$ & $<$ & $<$ & $<$ & $<$ \\
\hline 6 & $<$ & $<$ & $<$ & - & $>$ & $=$ & $<$ & $<$ & $<$ & - & - & $<$ & $<$ & $<$ & $<$ \\
\hline 7 & $<$ & - & $<$ & - & $>$ & $>$ & $=$ & $<$ & $<$ & $>$ & - & $<$ & $<$ & $<$ & $<$ \\
\hline 8 & $>$ & $>$ & - & $>$ & $>$ & $>$ & $>$ & $=$ & $>$ & $>$ & - & - & $<$ & - & - \\
\hline 9 & - & $>$ & - & - & $>$ & $>$ & $>$ & $<$ & $=$ & $>$ & - & - & $<$ & - & - \\
\hline 10 & $<$ & - & $<$ & $>$ & $<$ & $<$ & $<$ & $<$ & $=$ & $<$ & $<$ & $<$ & $<$ & $<$ \\
\hline 11 & - & - & - & $>$ & $>$ & - & - & - & - & $>$ & $=$ & - & $<$ & $<$ & - \\
\hline 12 & - & - & - & - & $>$ & $>$ & - & - & $>$ & - & $=$ & $<$ & $<$ & - \\
\hline 13 & $>$ & $>$ & $>$ & $>$ & $>$ & $>$ & $>$ & $>$ & $>$ & $>$ & $>$ & $=$ & $>$ & $>$ \\
\hline 14 & $>$ & - & $>$ & $>$ & $>$ & $>$ & - & - & $>$ & $>$ & $>$ & $<$ & $=$ & $>$ \\
\hline 15 & - & - & - & - & $>$ & $>$ & - & - & $>$ & - & - & $<$ & $<$ & $=$ \\
\hline 16 & - & $>$ & - & - & $>$ & $>$ & - & $>$ & $>$ & - & - & $<$ & - & $>$ \\
\hline 17 & - & - & - & - & $>$ & - & - & - & $>$ & $<$ & - & $<$ & $<$ & $<$ \\
\hline 18 & - & - & - & - & - & - & - & - & - & - & - & - & - \\
\hline 19 & - & - & - & - & - & - & - & - & - & - & - & - & - & - \\
\hline 20 & $>$ & - & $>$ & $>$ & $>$ & - & - & $>$ & - & $>$ & - & - & $>$ \\
\hline
\end{tabular}

(Continued on next page) 
Table 2 (Continued from previous page)

\begin{tabular}{|l|lllllllllllllll|}
\hline & 1 & 2 & 3 & 4 & 5 & 6 & 7 & 8 & 9 & 10 & 11 & 12 & 13 & 14 & 15 \\
\hline 21 & - & - & - & - & - & - & - & - & - & - & - & - & - & - & - \\
\hline 22 & - & - & - & - & - & - & - & - & - & - & - & - & - & - & - \\
\hline 23 & $>$ & $>$ & $>$ & $>$ & $>$ & $>$ & $>$ & $>$ & $>$ & $>$ & - & $>$ & - & - & $>$ \\
\hline 24 & - & - & - & - & - & - & - & - & - & - & - & - & - & - & - \\
\hline 25 & - & - & - & - & - & - & - & - & - & - & - & - & - & - & - \\
\hline 26 & $>$ & - & $>$ & $>$ & $>$ & $>$ & $>$ & - & - & $>$ & - & $>$ & $<$ & $<$ & - \\
\hline 27 & $>$ & $>$ & - & $>$ & $>$ & $>$ & $>$ & $>$ & $>$ & $>$ & - & - & $<$ & - & - \\
\hline 28 & - & - & - & $>$ & $>$ & - & - & - & - & $>$ & $<$ & - & $<$ & $<$ & - \\
\hline 29 & - & - & - & - & $>$ & $>$ & $>$ & - & - & $>$ & - & - & $<$ & $<$ & - \\
\hline 30 & - & - & - & - & - & - & - & - & - & - & - & - & $<$ & $<$ & - \\
\hline 31 & - & $>$ & - & - & $>$ & $>$ & $>$ & - & $>$ & $>$ & - & - & $<$ & - & - \\
\hline 32 & - & - & - & - & - & - & - & - & - & - & - & - & $<$ & - & - \\
\hline 33 & - & - & - & - & $>$ & - & - & - & - & $>$ & $<$ & - & $<$ & $<$ & - \\
\hline 34 & - & - & - & - & - & - & - & - & - & - & $<$ & - & $<$ & $<$ & - \\
\hline 35 & $<$ & - & $<$ & - & - & - & - & $<$ & - & - & - & - & $<$ & $<$ & - \\
\hline 36 & $<$ & - & $<$ & - & - & - & - & $<$ & - & - & - & - & $<$ & $<$ & - \\
\hline 37 & $<$ & - & $<$ & - & - & - & - & $<$ & - & - & - & - & $<$ & $<$ & - \\
\hline 38 & - & - & - & - & - & - & - & $<$ & - & - & - & - & $<$ & - & - \\
\hline 39 & $<$ & - & $<$ & $<$ & - & - & - & $<$ & - & - & $<$ & - & $<$ & $<$ & - \\
\hline 40 & - & - & - & - & - & - & - & $<$ & - & - & - & - & $<$ & - & - \\
\hline 41 & $<$ & - & $<$ & $<$ & - & - & - & $<$ & - & - & $<$ & - & $<$ & $<$ & - \\
\hline 52 & $>$ & $>$ & - & $>$ & $>$ & $>$ & $>$ & $<$ & $>$ & $>$ & - & - & $<$ & - & - \\
\hline 53 & - & $>$ & - & - & $>$ & $>$ & - & $<$ & $<$ & - & - & - & $<$ & - & - \\
\hline
\end{tabular}

Table 3 . Relations between the nonparametric intuitionistic fuzzy negations $\neg 16, \ldots, \neg 30$

\begin{tabular}{|c|ccccccccccccccc|}
\hline & 16 & 17 & 18 & 19 & 20 & 21 & 22 & 23 & 24 & 25 & 26 & 27 & 28 & 29 & 30 \\
\hline 1 & - & - & - & - & $<$ & - & - & $<$ & - & - & $<$ & $<$ & - & - & - \\
\hline 2 & $<$ & - & - & - & - & - & - & $<$ & - & - & - & $<$ & - & - & - \\
\hline 3 & - & - & - & - & $<$ & - & - & $<$ & - & - & $<$ & - & - & - & - \\
\hline 4 & - & - & - & - & $<$ & - & - & $<$ & - & - & $<$ & $<$ & $<$ & - & - \\
\hline 5 & $<$ & $<$ & - & - & $<$ & - & - & $<$ & - & - & $<$ & $<$ & $<$ & $<$ & - \\
\hline 6 & $<$ & - & - & - & $<$ & - & - & $<$ & - & - & $<$ & $<$ & - & $<$ & - \\
\hline 7 & $<$ & - & - & - & $<$ & - & - & $<$ & - & - & $<$ & $<$ & - & $<$ & - \\
\hline 8 & - & - & - & - & - & - & - & $<$ & - & - & - & $<$ & - & - & - \\
\hline 9 & $<$ & - & - & - & - & - & - & $<$ & - & - & - & $<$ & - & - & - \\
\hline 10 & $<$ & $<$ & - & - & $<$ & - & - & $<$ & - & - & $<$ & $<$ & $<$ & $<$ & - \\
\hline 11 & - & $>$ & - & - & - & - & - & - & - & - & - & - & $>$ & - & - \\
\hline
\end{tabular}


Table 3 (Continued from previous page)

\begin{tabular}{|l|lllllllllllllll|}
\hline & 16 & 17 & 18 & 19 & 20 & 21 & 22 & 23 & 24 & 25 & 26 & 27 & 28 & 29 & 30 \\
\hline 12 & - & - & - & - & $<$ & - & - & $<$ & - & - & $<$ & - & - & - & - \\
\hline 13 & $>$ & $>$ & - & - & - & - & - & - & - & - & $>$ & $>$ & $>$ & $>$ & $>$ \\
\hline 14 & - & $>$ & - & - & - & - & - & - & - & - & $>$ & - & $>$ & $>$ & $>$ \\
\hline 15 & $<$ & $>$ & - & - & $<$ & - & - & $<$ & - & - & - & - & - & - & - \\
\hline 16 & - & $>$ & - & - & - & - & - & $<$ & - & - & - & - & - & - & - \\
\hline 17 & $<$ & - & - & - & $<$ & - & - & $<$ & - & - & - & - & - & - & - \\
\hline 18 & - & - & - & $<$ & $<$ & - & $<$ & $<$ & - & $<$ & - & - & - & - & - \\
\hline 19 & - & - & $>$ & $=$ & $<$ & - & $<$ & $<$ & - & $<$ & - & - & - & - & $>$ \\
\hline 20 & - & $>$ & $>$ & $>$ & $=$ & - & - & $<$ & $>$ & $>$ & $>$ & - & $>$ & $>$ & $>$ \\
\hline 21 & - & - & - & - & - & $=$ & $<$ & $<$ & - & - & - & - & - & - & - \\
\hline 22 & - & - & $>$ & $>$ & - & $>$ & - & $<$ & - & - & - & - & - & - & $>$ \\
\hline 23 & $>$ & $>$ & $>$ & $>$ & $>$ & $>$ & $>$ & $=$ & $>$ & $>$ & $>$ & $>$ & $>$ & $>$ & $>$ \\
\hline 24 & - & - & - & - & $<$ & - & - & $<$ & $=$ & $<$ & - & - & - & - & - \\
\hline 25 & - & - & $>$ & $>$ & $<$ & - & - & $<$ & $>$ & $=$ & - & - & - & - & $>$ \\
\hline 26 & - & - & - & - & $<$ & - & - & $<$ & - & - & $=$ & - & $>$ & - & - \\
\hline 27 & - & - & - & - & - & - & - & $<$ & - & - & - & $=$ & - & - & - \\
\hline 28 & - & - & - & - & $<$ & - & - & $<$ & - & - & $<$ & - & $=$ & - & - \\
\hline 29 & - & - & - & - & $<$ & - & - & $<$ & - & - & - & - & - & $=$ & $>$ \\
\hline 30 & - & - & - & $<$ & $<$ & - & $<$ & $<$ & - & $<$ & - & - & - & $<$ & $=$ \\
\hline 31 & - & - & - & - & - & - & - & $<$ & - & - & - & - & - & - & - \\
\hline 32 & - & - & - & - & - & - & $<$ & $<$ & - & - & - & - & - & - & - \\
\hline 33 & - & - & - & - & $<$ & - & - & $<$ & - & - & - & - & - & - & - \\
\hline 34 & - & - & - & - & $<$ & - & - & $<$ & - & $<$ & - & - & - & - & - \\
\hline 35 & - & - & - & - & $<$ & - & - & $<$ & - & - & $<$ & $<$ & - & - & - \\
\hline 36 & - & - & - & - & $<$ & - & - & $<$ & - & - & $<$ & $<$ & - & - & - \\
\hline 37 & - & - & - & - & $<$ & - & - & $<$ & - & - & $<$ & $<$ & - & - & - \\
\hline 38 & - & - & - & - & - & - & - & $<$ & - & - & - & $<$ & - & - & - \\
\hline 39 & - & - & - & - & $<$ & - & - & $<$ & - & - & $<$ & $<$ & $<$ & - & - \\
\hline 40 & - & - & - & - & - & - & - & $<$ & - & - & - & $<$ & - & - & - \\
\hline 41 & - & - & - & - & $<$ & - & - & $<$ & - & - & $<$ & $<$ & $<$ & - & - \\
\hline 52 & - & - & - & - & - & - & - & $<$ & - & - & - & $<$ & - & - & - \\
\hline 53 & $<$ & - & - & - & - & - & - & $<$ & - & - & - & $<$ & - & - & - \\
\hline
\end{tabular}


Table 4. Relations between the nonparametric intuitionistic fuzzy negations $\neg_{31}, \ldots, \neg_{41}, \neg_{52}, \neg_{53}$

\begin{tabular}{|c|c|c|c|c|c|c|c|c|c|c|c|c|c|}
\hline & 31 & 32 & 33 & 34 & 35 & 36 & 37 & 38 & 39 & 40 & 41 & 52 & 53 \\
\hline 1 & - & - & - & - & $>$ & $>$ & $>$ & - & $>$ & - & $>$ & $<$ & - \\
\hline 2 & $<$ & - & - & - & - & - & - & - & - & - & - & $<$ & $<$ \\
\hline 3 & - & - & - & - & $>$ & $>$ & $>$ & - & $>$ & - & $>$ & - & - \\
\hline 4 & - & - & - & - & - & - & - & - & $>$ & - & $>$ & $<$ & - \\
\hline 5 & $<$ & - & $<$ & - & - & - & - & - & - & - & - & $<$ & $<$ \\
\hline 6 & $<$ & - & - & - & - & - & - & - & - & - & - & $<$ & $<$ \\
\hline 7 & $<$ & - & - & - & - & - & - & - & - & - & - & $<$ & - \\
\hline 8 & - & - & - & - & $>$ & $>$ & $>$ & $>$ & $>$ & $>$ & $>$ & $>$ & $>$ \\
\hline 9 & $<$ & - & - & - & - & - & - & - & - & - & - & $<$ & $>$ \\
\hline 10 & $<$ & - & $<$ & - & - & - & - & - & - & - & - & $<$ & - \\
\hline 11 & - & - & $>$ & $>$ & - & - & - & - & $>$ & - & $>$ & - & - \\
\hline 12 & - & - & - & - & - & - & - & - & - & - & - & - & - \\
\hline 13 & $>$ & $>$ & $>$ & $>$ & $>$ & $>$ & $>$ & $>$ & $>$ & $>$ & $>$ & $>$ & $>$ \\
\hline 14 & - & - & $>$ & $>$ & $>$ & $>$ & $>$ & - & $>$ & - & $>$ & - & - \\
\hline 15 & - & - & - & - & - & - & - & - & - & - & - & - & - \\
\hline 16 & - & - & - & - & - & - & - & - & - & - & - & - & $>$ \\
\hline 17 & - & - & - & - & - & - & - & - & - & - & - & - & - \\
\hline 18 & - & - & - & - & - & - & - & - & - & - & - & - & - \\
\hline 19 & - & - & - & - & - & - & - & - & - & - & - & - & - \\
\hline 20 & - & - & $>$ & $>$ & $>$ & $>$ & $>$ & - & $>$ & - & $>$ & - & - \\
\hline 21 & - & - & - & - & - & - & - & - & - & - & - & - & - \\
\hline 22 & - & $>$ & - & - & - & - & - & - & - & - & - & - & - \\
\hline 23 & $>$ & $>$ & $>$ & $>$ & $>$ & $>$ & $>$ & $>$ & $>$ & $>$ & $>$ & $>$ & $>$ \\
\hline 24 & - & - & - & - & - & - & - & - & - & - & - & - & - \\
\hline 25 & - & - & - & $>$ & - & - & - & - & - & - & - & - & - \\
\hline 26 & - & - & - & - & $>$ & $>$ & $>$ & - & $>$ & - & $>$ & - & - \\
\hline 27 & - & - & - & - & $>$ & $>$ & $>$ & $>$ & $>$ & $>$ & $>$ & $>$ & $>$ \\
\hline 28 & - & - & - & - & - & - & - & - & $>$ & - & $>$ & - & - \\
\hline 29 & - & - & - & - & - & - & - & - & - & - & - & - & - \\
\hline 30 & - & - & - & - & - & - & - & - & - & - & - & - & - \\
\hline 31 & $=$ & $>$ & - & - & - & - & - & - & - & - & - & - & $>$ \\
\hline 32 & $<$ & $=$ & - & - & - & - & - & - & - & - & - & - & - \\
\hline 33 & - & - & $=$ & $>$ & - & - & - & - & - & - & - & - & - \\
\hline 34 & - & - & $<$ & $=$ & - & - & - & - & - & - & - & - & - \\
\hline 35 & - & - & - & - & $=$ & $>$ & $<$ & - & $>$ & $<$ & - & $<$ & - \\
\hline 36 & - & - & - & - & $<$ & $=$ & $<$ & $<$ & $>$ & $<$ & - & $<$ & - \\
\hline
\end{tabular}


Table 4 (Continued from previous page)

\begin{tabular}{|l|ccccccccccccc|}
\hline & 31 & 32 & 33 & 34 & 35 & 36 & 37 & 38 & 39 & 40 & 41 & 52 & 53 \\
\hline 37 & - & - & - & - & $>$ & $>$ & $=$ & - & $>$ & $<$ & $>$ & $<$ & - \\
\hline 38 & - & - & - & - & - & $>$ & - & $=$ & $>$ & $<$ & - & - & - \\
\hline 39 & - & - & - & - & $<$ & $<$ & $<$ & $<$ & $=$ & $<$ & $<$ & $<$ & - \\
\hline 40 & - & - & - & - & $>$ & $>$ & $>$ & $>$ & $>$ & $=$ & $>$ & - & - \\
\hline 41 & - & - & - & - & - & - & $<$ & - & $>$ & $<$ & $=$ & $<$ & - \\
\hline 52 & - & - & - & - & $>$ & $>$ & $>$ & - & $>$ & - & $>$ & $=$ & $>$ \\
\hline 53 & $<$ & - & - & - & - & - & - & - & - & - & - & $<$ & $=$ \\
\hline
\end{tabular}

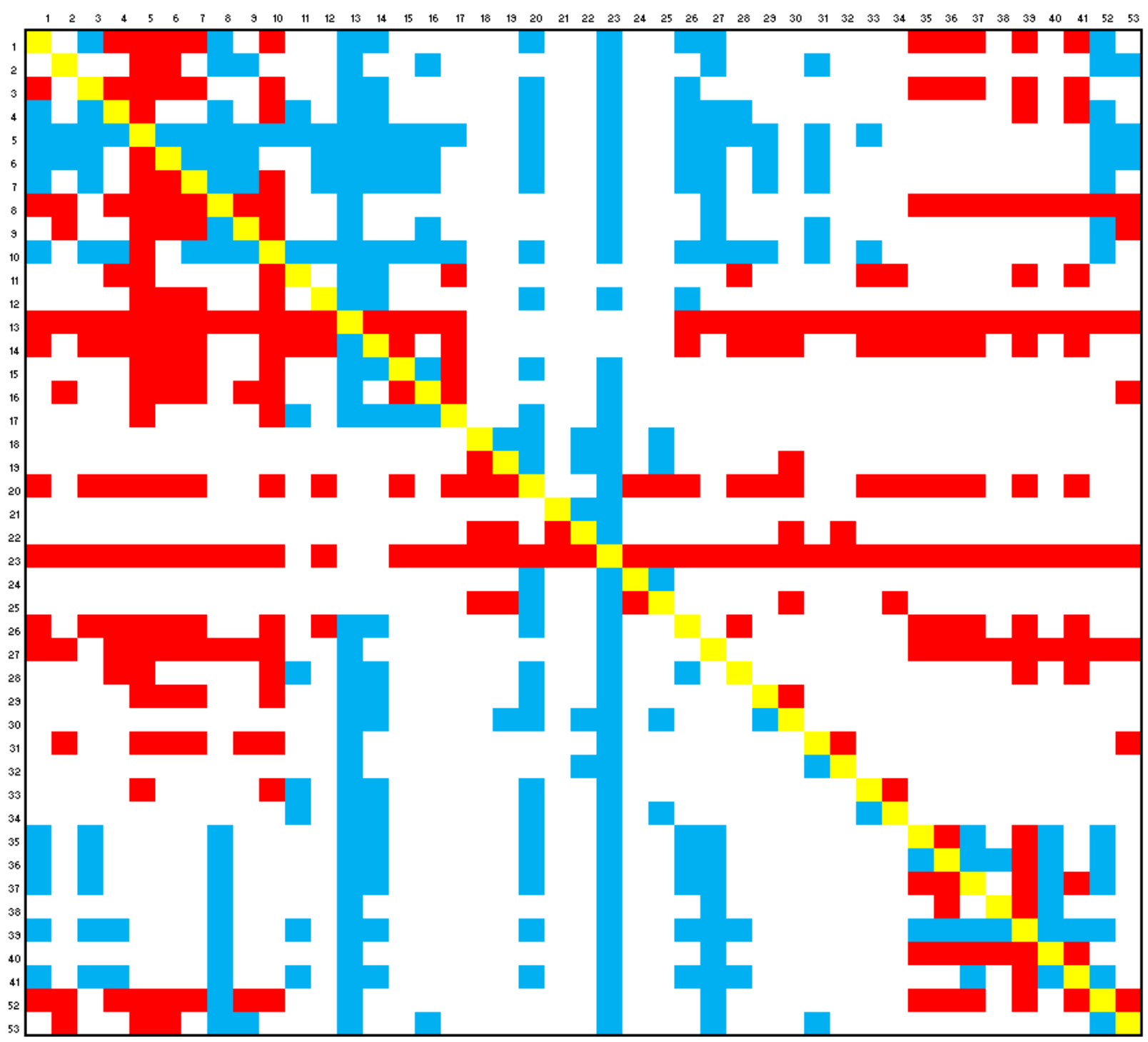

Figure 1. Visualization of the Tables 2 to 4.

Red cells are $>$, blue cells are $<$, yellow cells are $=$, white cells are - .

The relationships between the negations and implications are shown in Table 5. Following [17], in Table 5 we show the relationships between the negations and implications, determining 
the implications that generate the different negations. We can see that some negations, e.g., $\neg_{1}, \neg_{2}, \neg_{4}, \neg_{5}, \neg_{8}$ are generated from a lot of different implications, while for other negations there are unique implications that generate them.

Table 5. Relationships between negations and implications

\begin{tabular}{|c|c|}
\hline$\neg 1$ & $\begin{array}{l}\rightarrow_{1}, \rightarrow_{4}, \rightarrow_{5}, \rightarrow_{6}, \rightarrow_{7}, \rightarrow_{10}, \rightarrow_{13}, \rightarrow_{61}, \rightarrow_{63}, \rightarrow_{64}, \rightarrow_{66}, \rightarrow_{67}, \rightarrow_{68}, \rightarrow_{69}, \\
\rightarrow_{70}, \rightarrow_{71}, \rightarrow_{72}, \rightarrow_{73}, \rightarrow_{78}, \rightarrow_{80}, \rightarrow_{124}, \rightarrow_{125}, \rightarrow_{127}, \rightarrow_{166}, \rightarrow_{186}, \rightarrow_{192}\end{array}$ \\
\hline$\neg 2$ & $\begin{array}{l}\rightarrow_{2}, \rightarrow_{3}, \rightarrow_{8}, \rightarrow_{11}, \rightarrow_{16}, \rightarrow_{20}, \rightarrow_{31}, \rightarrow_{32}, \rightarrow_{37}, \rightarrow_{40}, \rightarrow_{41}, \rightarrow_{42} \\
\rightarrow_{172}, \rightarrow_{173}, \rightarrow_{181}, \rightarrow_{182}, \rightarrow_{183}\end{array}$ \\
\hline$\neg_{3}$ & $\rightarrow_{9}, \rightarrow_{17}, \rightarrow_{21}$ \\
\hline$\neg_{4}$ & $\begin{array}{l}\rightarrow_{12}, \rightarrow_{18}, \rightarrow_{22}, \rightarrow_{46}, \rightarrow_{49}, \rightarrow_{50}, \rightarrow_{51}, \rightarrow_{53}, \rightarrow_{54}, \rightarrow_{91}, \rightarrow_{93}, \rightarrow_{94}, \\
\rightarrow_{95}, \rightarrow_{96}, \rightarrow_{98}, \rightarrow_{134}, \rightarrow_{135}, \rightarrow_{137}, \rightarrow_{169}, \rightarrow_{170}, \rightarrow_{179}, \rightarrow_{180}, \rightarrow_{187}, \rightarrow_{189}, \\
\rightarrow_{190}, \rightarrow_{193}, \rightarrow_{197}, \rightarrow_{198}\end{array}$ \\
\hline$\neg_{5}$ & $\begin{array}{l}\rightarrow_{14}, \rightarrow_{15}, \rightarrow_{19}, \rightarrow_{23}, \rightarrow_{47}, \rightarrow_{48}, \rightarrow_{52}, \rightarrow_{55}, \rightarrow_{56}, \rightarrow_{57}, \rightarrow_{171}, \rightarrow_{174}, \\
\rightarrow_{175}, \rightarrow_{184}, \rightarrow_{185}\end{array}$ \\
\hline$\neg 6$ & $\rightarrow_{24}, \rightarrow_{26}, \rightarrow_{27}, \rightarrow_{65}$ \\
\hline$\neg 7$ & $\rightarrow_{25}, \rightarrow_{28}, \rightarrow_{29}, \rightarrow_{62}$ \\
\hline$\neg 8$ & $\begin{array}{l}\rightarrow_{30}, \rightarrow_{33}, \rightarrow_{34}, \rightarrow_{35}, \rightarrow_{36}, \rightarrow_{38}, \rightarrow_{39}, \rightarrow_{76}, \rightarrow_{82}, \rightarrow_{84}, \rightarrow_{85}, \rightarrow_{86}, \rightarrow_{87}, \\
\rightarrow_{89}, \rightarrow_{129}, \rightarrow_{130}, \rightarrow_{132}, \rightarrow_{167}, \rightarrow_{168}, \rightarrow_{177}, \rightarrow_{178}, \rightarrow_{188}, \rightarrow_{194}, \rightarrow_{195}, \rightarrow_{196}\end{array}$ \\
\hline$\neg 9$ & $\rightarrow_{43}, \rightarrow_{44}, \rightarrow_{45}, \rightarrow_{83}$ \\
\hline$\neg 10$ & $\rightarrow_{58}, \rightarrow_{59}, \rightarrow_{60}, \rightarrow_{92}$ \\
\hline$\neg 11$ & $\rightarrow_{74}, \rightarrow_{97}$ \\
\hline$\neg 12$ & $\rightarrow 75$ \\
\hline$\neg 13$ & $\rightarrow_{77}, \rightarrow_{88}$ \\
\hline$\neg 14$ & $\rightarrow_{79}$ \\
\hline$\neg 15$ & $\rightarrow_{81}$ \\
\hline$\neg 16$ & $\rightarrow_{90}$ \\
\hline$\neg 17$ & $\rightarrow_{99}$ \\
\hline$\neg 18$ & $\rightarrow_{100}$ \\
\hline$\neg 19$ & $\rightarrow_{101}$ \\
\hline$\neg_{20}$ & $\rightarrow_{102}, \rightarrow_{108}$ \\
\hline$\neg_{21}$ & $\rightarrow_{103}$ \\
\hline$\neg_{22}$ & $\rightarrow_{104}$ \\
\hline$\neg_{23}$ & $\rightarrow_{105}$ \\
\hline$\neg_{24}$ & $\rightarrow_{106}$ \\
\hline$\neg 25$ & $\rightarrow_{107}$ \\
\hline$\neg 26$ & $\rightarrow_{109}, \rightarrow_{110}, \rightarrow_{111}, \rightarrow_{112}, \rightarrow_{113}$ \\
\hline$\neg 27$ & $\rightarrow_{114}, \rightarrow_{115}, \rightarrow_{116}, \rightarrow_{117}, \rightarrow_{118}$ \\
\hline$\neg 28$ & $\rightarrow_{119}, \rightarrow_{120}, \rightarrow_{121}, \rightarrow_{122}, \rightarrow_{123}$ \\
\hline
\end{tabular}

(Continued on next page) 
Table 5 (Continued from previous page)

\begin{tabular}{|l|l|}
\hline$\neg_{29}$ & $\rightarrow_{126}$ \\
\hline$\neg_{30}$ & $\rightarrow_{128}$ \\
\hline$\neg_{31}$ & $\rightarrow_{131}$ \\
\hline$\neg_{32}$ & $\rightarrow_{133}$ \\
\hline$\neg_{33}$ & $\rightarrow_{136}$ \\
\hline$\neg_{34}$ & $\rightarrow_{138}$ \\
\hline$\neg_{35}$ & $\rightarrow_{139}$ \\
\hline$\neg_{36}$ & $\rightarrow_{140}$ \\
\hline$\neg_{37}$ & $\rightarrow_{141}$ \\
\hline$\neg_{38}$ & $\rightarrow_{142}, \rightarrow_{143}$ \\
\hline$\neg_{39}$ & $\rightarrow_{144}, \rightarrow_{145}$ \\
\hline$\neg_{40}$ & $\rightarrow_{146}, \rightarrow_{147}$ \\
\hline$\neg_{41}$ & $\rightarrow_{148,} \rightarrow_{149}$ \\
\hline$\neg_{42, \lambda}$ & $\rightarrow_{150}$ \\
\hline$\neg_{43, \gamma}$ & $\rightarrow_{151}$ \\
\hline$\neg_{44, \alpha, \beta}$ & $\rightarrow_{152}$ \\
\hline$\neg_{45, \varepsilon, \eta}$ & $\rightarrow_{153}$ \\
\hline$\neg_{46, \lambda}$ & $\rightarrow_{154, \lambda}, \rightarrow_{155, \lambda}$ \\
\hline$\neg_{47, \lambda}$ & $\rightarrow_{156, \lambda}, \rightarrow_{157, \lambda}$ \\
\hline$\neg_{48, \gamma}$ & $\rightarrow_{158, \gamma}, \rightarrow_{159, \gamma}$ \\
\hline$\neg_{49, \gamma}$ & $\rightarrow_{160, \gamma}, \rightarrow_{161, \gamma}$ \\
\hline$\neg_{50, \alpha, \beta}$ & $\rightarrow_{162, \alpha, \beta}, \rightarrow_{163, \alpha, \beta}$ \\
\hline$\neg_{51, \alpha, \beta}$ & $\rightarrow_{164, \alpha, \beta}, \rightarrow_{165, \alpha, \beta}$ \\
\hline$\neg_{52}$ & $\rightarrow_{176}$ \\
\hline$\neg_{53}$ & $\rightarrow_{191}$ \\
\hline & \\
\hline
\end{tabular}

\section{Conclusion: Ideas for the future}

Our idea for the present paper is a second step (after this, described in [4]) of a more general research. Using the results from Table 1, we will construct, by analogy with [14], oriented graphs with vertices corresponding to the different negation and oriented arcs with directions determined by the symbols of the Table 1. After this, we will search those elements of the graphs that satisfy the axioms for negations (and implications), checked in [17].

Finally, we will preserve only the negations exhibiting the best modelling properties, that are related to the implications with the best modelling properties, and for them, we will offer a new numeration, different from the one adopted so far in the literature. In this way, we will address an important question that we have been facing for the last 15 years, regarding the comparability, rangeability and modelling applicability of the numerous distinct implications and negations over intuitionistic fuzzy sets. 


\section{Acknowledgements}

This research was funded by Bulgarian National Science Fund, Grant Ref. No. KP-06-N22/1/2018 "Theoretical research and applications of InterCriteria Analysis".

\section{References}

[1] Angelova, N. (2019). IFSTool - Software for intuitionistic fuzzy sets - Necessity, Possibility and Circle operators. Advances in Intelligent Systems and Computing, issue:1081, Publisher:Springer, 76-81.

[2] Angelova, N., \& Atanassov, K. (2015). Intuitionistic Fuzzy Implications and the Axioms of Intuitionistic Logic. In: Proc. of the 9th Conference of the European Society for Fuzzy Logic and Technology (EUSFLAT), 30.06-03.07.2015, Gijon, Spain, 1578-1584.

[3] Angelova, N., \& Atanassov, K. (2016). Intuitionistic Fuzzy Implications and Klir-Yuan's Axioms. Novel Developments in Uncertainty Representation and Processing. Advances in Intuitionistic Fuzzy Sets and Generalized Nets, Advances in Intelligent Systems and Computing, 401. Atanassov, K.T., Castillo, O., Kacprzyk, J., Krawczak, M., Melin, P., Sotirov, S., Sotirova, E., Szmidt, E., De Tré, G., Zadrożny, S. (Eds.), 97-110.

[4] Angelova, N., \& Atanassov, K. (2021). Research on intuitionistic fuzzy implications. Notes on Intuitionistic Fuzzy Sets, 27(2), 20-93.

[5] Angelova, N., Marinov, E., \& Atanassov, K. (2015). Intuitionistic fuzzy implications and Kolmogorov's and Łukasiewisz-Tarski's axioms of logic. Notes on Intuitionistic Fuzzy Sets, 21(2), 35-42.

[6] Atanassov, K. (1988). Two variants of intuitonistic fuzzy propositional calculus. Preprint IM-MFAIS-5-88, Sofia.

[7] Atanassov, K. (2005). On some intuitionistic fuzzy negations. Proc. of the First Int. Workshop on IFSs, Banska Bystrica, 22 Sept. 2005. Notes on Intuitionistic Fuzzy Sets, 11(6), 13-20.

[8] Atanassov, K. (2006). A new intuitionistic fuzzy implication from a modal type. Advanced Studies on Contemporary Mathematics, 12 (1), 117-122.

[9] Atanassov, K. (2006). On eight new intuitionistic fuzzy implications. Proc. of 3rd Int. IEEE Conf. “Intelligent Systems” ISO6, London, 4-6 Sept. 2006, 741-746.

[10] Atanassov, K. (2006). On some intuitionistic fuzzy implications. Comptes Rendus de l'Academie bulgare des Sciences, 59(1), 19-24.

[11] Atanassov, K. (2008). On intuitionistic fuzzy implication $\rightarrow^{\varepsilon}$ and intuitionistic fuzzy negation $\neg^{\varepsilon}$. Issues in Intuitionistic Fuzzy Sets and Generalized Nets, 6, 6-19. 
[12] Atanassov, K. (2008). Intuitionistic fuzzy implication $\rightarrow^{\varepsilon, \eta}$ and intuitionistic fuzzy negation $\neg^{\varepsilon, \eta}$. Developments in Fuzzy Sets, Intuitionistic Fuzzy Sets, Generalized Nets and Related Topics, 1, 1-10.

[13] Atanassov, K. (2011). Second Zadeh's intuitionistic fuzzy implication. Notes on Intuitionistic Fuzzy Sets, 17(3), 11-14.

[14] Atanassov, K. (2012). On Intuitionistic Fuzzy Sets Theory, Springer, Berlin.

[15] Atanassov, K. (2015). On a new intuitionistic fuzzy implication. In: Proc of the 9th Conference of the European Society for Fuzzy Logic and Technology (EUSFLAT), 30.0603.07.2015, Gijon, Spain, 1592-1597.

[16] Atanassov, K. (2016). On intuitionistic fuzzy implications. Issues in Intuitionistic Fuzzy Sets and Generalized Nets, 12, 1-19.

[17] Atanassov, K. (2017). Intuitionistic Fuzzy Logics, Springer, Cham.

[18] Atanassov, K (2021). Third Zadeh's Intuitionistic Fuzzy Implication. Mathematics, 9(6), 619.

[19] Atanassov, K., \& Angelova, N. (2021). Modifications of the Third Zadeh's intuitionistic fuzzy implication. Notes on Intuitionistic Fuzzy Sets, 27(1), 9-23.

[20] Atanassov, K., \& Angelova, N. (2016). Properties of intuitionistic fuzzy implications and negations. Notes on Intuitionistic Fuzzy Sets, 22(3), 25-33.

[21] Atanassov, K., Angelova, N.\& Atanassova, V. (2021). On an Intuitionistic Fuzzy Form of the Goguen's Implication. Mathematics, 9(6), 676.

[22] Atanassov, K., \& Dimitrov, D. (2010). Intuitionistic fuzzy implications and axioms for implications. Notes in Intuitionistic Fuzzy Sets, 16(1), 10-20.

[23] Atanassov, K., Ribagin, S., Doukovska, L., \& Atanassova, V. (2017). Intuitionistic fuzzy implication $\rightarrow{ }_{190}$. Notes on Intuitionistic Fuzzy Sets, 23(4), 79-83.

[24] Atanassov, K., \& Szmidt, E. (2014). Remark on intuitionistic fuzzy implication $\rightarrow^{\varepsilon, \eta}$. Issues in Intuitionistic Fuzzy Sets and Generalized Nets, 11, 9-14.

[25] Atanassov, K., Szmidt, E., \& Kacprzyk, J. (2013). On intuitionistic fuzzy pairs, Notes on Intuitionistic Fuzzy Sets, 19(3), 1-13.

[26] Atanassov, K., Szmidt, E. \& Kacprzyk, J. (2015). On Fodor's type of intuitionistic fuzzy implication and negation. Notes on Intuitionistic Fuzzy Sets, 21(2), 25-34.

[27] Atanassov, K., Szmidt, E., \& Kacprzyk, J. (2016). New Fodor's Type Of Intuitionistic Fuzzy Implication and Negation. Notes on Intuitionistic Fuzzy Sets, 22(3), 1-8. 
[28] Atanassov, K., Szmidt, E., Kacprzyk, J., \& Angelova, N. (2019). Intuitionistic fuzzy implications revisited. Part 1. Notes on Intuitionistic Fuzzy Sets, 25(3), 71-78.

[29] Atanassov, K., \& Trifonov, T. (2005). On a new intuitionistic fuzzy implication of Godel's type. Proceedings of the Jangjeon Mathematical Society, 8(2), 147-152.

[30] Atanassov, K., \& Trifonov, T. (2006). Two new intuitionistic fuzzy implications. Advanced Studies on Contemporary Mathematics, 13(1), 69-74.

[31] Atanassova, L. (2008). On an intuitionistic fuzzy implication from Kleene-Dienes type. Proceedings of the Jangjeon Mathematical Society, 11(1), 69-74.

[32] Atanassova, L. (2008). Modifications of an intuitionistic fuzzy implication from KleeneDienes type. Advanced Studies in Contemporary Mathematics, 16 (2), 155-160.

[33] Atanassova, L. (2008). New modifications of an intuitionistic fuzzy implication from Kleene-Dienes type. Part 2. Annual of Section "Informatics”, 1, 59-64.

[34] Atanassova, L. (2009). New modifications of an intuitionistic fuzzy implication from Kleene-Dienes type. Part 3. Advanced Studies in Contemporary Mathematics, 18 (1), 33-40.

[35] Atanassova, L. (2009). A new intuitionistic fuzzy implication. Cybernetics and Information Technologies, 9(2), 21-25.

[36] Atanassova, L. (2009). On some properties of intuitionistic fuzzy negation $\neg @$. Notes on Intuitionistic Fuzzy Sets, 15(1), 32-35.

[37] Atanassova, L. (2012). On two modifications of the intuitionistic fuzzy implication $\rightarrow @$. Notes on Intuitionistic Fuzzy Sets, 18(2), 26-30

[38] Atanassova, L. (2013). On the modal form of the intuitionistic fuzzy implications $\rightarrow_{@}^{\prime}$ and $\rightarrow{ }_{@}^{\prime \prime}$. Issues in Intuitionistic Fuzzy Sets and Generalized Nets, 10, 5-11.

[39] Atanassova, L. (2013). On the intuitionistic fuzzy form of the classical implication $(A \rightarrow$ $B) \vee(B \rightarrow A)$. Notes on Intuitionistic Fuzzy Sets, 19(4), 15-18.

[40] Atanassova, L. (2014). Remark on the intuitionistic fuzzy forms of two classical logic axioms. Part 1. Annual of Section "Informatics", 7, 24-27.

[41] Atanassova, L. (2014). Remark on the intuitionistic fuzzy forms of two classical logic axioms. Part 2. Notes on Intuitionistic Fuzzy Sets, 20(4), 10-13.

[42] Atanassova, L. (2015). Remark on Dworniczak's intuitionistic fuzzy implications. Part 1. Notes on Intuitionistic Fuzzy Sets, 21(3), 18-23.

[43] Atanassova, L. (2015). Remark on Dworniczak's intuitionistic fuzzy implications. Part 2. Issues in Intuitionistic Fuzzy Sets and Generalized Nets, 12, 61-67. 
[44] Atanassova, L. (2016). Remark on Dworniczak's intuitionistic fuzzy implications. Part 3. Notes on Intuitionistic Fuzzy Sets, 22(1), 1-6.

[45] Atanassova, L. (2017). Properties of the intuitionistic fuzzy implication $\rightarrow_{189}$. Notes on Intuitionistic Fuzzy Sets, 23(4), 10-14.

[46] Dworniczak, P. (2010). Some remarks about the L. Atanassova's paper "A new intuitionistic fuzzy implication", Cybernetics and Information Technologies, 10(3), 3-9.

[47] Dworniczak, P. (2010). On one class of intuitionistic fuzzy implications. Cybernetics and Information Technologies, 10(4), 13-21.

[48] Dworniczak, P. (2011). On some two-parametric intuitionistic fuzzy implication. Notes on Intuitionistic Fuzzy Sets, 17(2), 8-16.

[49] Klir, G., \& Yuan, B. (1995). Fuzzy Sets and Fuzzy Logic. Prentice Hall, New Jersey.

[50] Szmidt, E., Kacprzyk, J., \& Atanassov, K. (2015). Properties of Fodor's intuitionistic fuzzy implication and negation. Notes on Intuitionistic Fuzzy Sets, 21(4), 6-12.

[51] Szmidt, E., Kacprzyk, J., \& Atanassov, K. (2015). Modal forms of Fodor's type of intuitionistic fuzzy implication. Notes on Intuitionistic Fuzzy Sets, 21(5), 1-5.

[52] Vassilev, P., \& Atanassov, K. (2019). Extensions and Modifications of Intuitionistic Fuzzy Sets. "Prof. Marin Drinov" Academic Publishing House, Sofia. 